\title{
Comparing Skin Grafts Harvested From Wrist With Those Harvested From Groin Region in Patients With Fingertip Injury: A Randomized Clinical Trial
}

\author{
Hamid Namazi ${ }^{1}$; Reza Sharifzadeh ${ }^{2}$; Behdad Eskandari Sani ${ }^{1}$; Farzam Mokarami ${ }^{2, *}$ \\ ${ }^{1}$ Department of Orthopedic Surgery, Nemazee Hospital, Shiraz University of Medical Sciences, Shiraz, IR Iran \\ 2 Department of Orthopedic Surgery, Imam Reza Hospital, AJA University of Medical Sciences, Tehran, IR Iran \\ *Corresponding author: Farzam Mokarami, Department of Orthopedic Surgery, Imam Reza Hospital, AJA University of Medical Sciences, Tehran, IR Iran. Tel: +98-2185955616, Fax: \\ +98-2188028931, E-mail: farzam_m2001@yahoo.com
}

Received: August 20, 2014; Revised: September 15, 2014; Accepted: November 22, 2014

Background: Fingertip injuries are among the most prevalent hand injuries. The fingertip has important sensory and functional role. When the size of fingertip skin defect is large, full thickness skin grafting is necessary. There are several donor sites for obtaining skin graft; however, there is no study concerning the best region of harvesting skin graft compatible with the fingertip.

Objectives: This study was designed to compare the skin grafts harvested from wrist and groin for fingertip skin loss.

Patients and Methods: A total of 72 patients in need of skin grafting were randomized to two group of 36 to receive skin graft harvested from the wrist or from the groin regions. The patients included 71 males and only one female patient who was in the wrist harvested group. All the patients were operated by one technique. The minimum follow-up period was 18 months. The grafted skin was evaluated clinically regarding two-point discrimination (TPD), light touch, ulcer, graft contracture, hair growth on the grafted skin, and color difference between the graft and recipient site, which was evaluated with photo analyzer in Photoshop software.

Results: The grafted skins were compared between groups. Clinically, TPD and light touch were better (lower thresholds) in the wrist group $(\mathrm{P}<0.05)$. Moreover, The amount of skin contracture, color difference between grafted skin and recipient site, and hair follicle counts were less in the wrist group $(\mathrm{P}<0.001)$. In both groups, there were no ulcer and fissuring.

Conclusions: For fingertip injures, full thickness skin grafts harvested from the wrist have significantly better cosmetic and functional results including better TPD and light touch, less hair follicles, less color difference, and less contracture.

Keywords: Skin Grafting; Fingertip; Donor Site; Skin

\section{Background}

Fingertip injuries are among the most prevalent hand injuries and immediate management of these injuries is imperative $(1,2)$. When skin loss is more than half of the pulp size, coverage with skin graft leads to faster recovery with less morbidity (1). Full-thickness skin grafts supply better protection and sensation as well as less contracture than split thickness skin graft does $(1,3-5)$. The goal is performing full thickness skin graft from inconspicuous donor sites that provide an excellent clinical and photographic match in terms of color, texture, thickness, and sebaceous quality. Yet, there is no study concerning the clinical difference among these skin grafts outcomes.

\section{Objectives}

This randomized clinical trial has been structured to compare the quality of skin grafts harvested from wrist flexor crease with those harvested from groins for fingertip skin defects through evaluating two-point discrimination (TPD), light touch, ulcer, graft contracture, hair growth on the grafted skin, and color difference between the grafted skin and surrounding skin.

\section{Patients and Methods}

This multicenter randomized clinical trial was conducted in accordance with the ethical principles of Helsinki Declaration and was approved by the Ethical Committee of Shiraz University of Medical Sciences (Project No, ct.p.91.2611; IRCT registration ID, IRCT2014110413259N1. All the patients or their guardians signed the written informed consent of the study procedure. All of the operations were done from August 2011 to April 2012 in two medical centers; Nemazee Hospital, Shiraz, Iran, and Imam Reza Hospital, Tehran, Iran, with a minimum follow-up period of 18 months.

\subsection{Patients' Eligibility}

A total of 72 patients with acute traumatic fingertip injuries requiring skin grafts were evaluated for eligibility. The eligibility criteria were as follows: aging 20 to70 years, normal sensation and judgment, being nonsmoker, no neuropathic disorders including diabetes, autoimmune diseases (e.g. scleroderma), congestive heart failure, chronic liver diseases, chronic renal failure, and peripheral vascular diseases. 
Table 1. Basic Characteristics of Patients With Fingertip Injuries Undergoing Skin Grafting

\begin{tabular}{lcc}
\hline & \multicolumn{2}{c}{ Region of Harvested Skin Graft } \\
\cline { 2 - 3 } & Wrist, $\mathbf{N}=\mathbf{3 6}$ & Groin, $\mathbf{N}=\mathbf{3 6}$ \\
\hline $\begin{array}{l}\text { Sex, male to female } \\
\text { ratio }\end{array}$ & $35 / 1$ & $36 / 0$ \\
\hline $\begin{array}{l}\text { Age, mean } \pm \text { SD, } \mathbf{y} \\
\begin{array}{l}\text { Surgery time, } \\
\text { mean } \pm \text { SD, min }\end{array}\end{array}$ & $38 \pm 6.8$ & $34 \pm 6.2$ \\
$\begin{array}{l}\text { Body mass index, } \\
\text { mean } \pm \mathbf{S D}, \mathbf{~ k g} / \mathbf{m}^{\mathbf{2}}\end{array}$ & $21 \pm 7.2$ & $27 \pm 6.3$ \\
\hline
\end{tabular}

\subsection{Sample Size and Randomization}

Considering $40 \%$ difference in variables, power of $80 \%$, and of 0.05 , sample size of at least 36 patients was calculated according to the previous studies (6). In order to increase the study power and reproducibility, we enrolled 72 eligible patients with fingertip full-thickness skin injuries and randomly allocated them to two groups of 36, ie, wrist group and groin group, by Research Randomizer Program (7). All patients had fingertip skin loss of more than $50 \%$ of the pulp area at index, middle, or ring fingers (Table 1 ).

\subsection{Operation Techniques}

Because it was a multicenter study, we strictly stuck to the technique of harvesting grafts. Briefly, we harvested the adjusted skin graft from the wrist or groin in elliptical shape so that it correspond to the size of the lost region in fingertips and tried to remove the subcutaneous fat completely to increase the chance of successful grafting. No meshing procedure was used. Grafts were fixed with absorbable 4.0 (Vicryl, Ethicon Inc, Somerville, New Jersey, USA) running sutures. Then dressing was applied on by four-layered sterile gauze in a moderately tight fashion and due to the low risk of hematoma formation, the grafts were seen after five to seven days.

\subsection{Outcomes and Data Analysis}

At least six months after the operation, the patients were evaluated clinically and photographically through TPD, light touch, existence of hair on graft, contracture and ulceration of the grafted skin, and color difference with recipient site.

For evaluation of TPD, we used paper clips (DellonMcKinnon Disk-Criminator, Fabrication Enterprises Inc, White Plains, New York, USA) fixed in definite distances $(2,3,4,5,6,7,8$, and $9 \mathrm{~mm}$ ) (Figure 1) (8).

Patients were asked to close their eyes and we checked the patients' contralateral healthy fingertip with $2-\mathrm{mm}$ device to determine whether the patient recognized needles as two points. Then we applied it on the grafted skin and asked the patient about points discriminating.

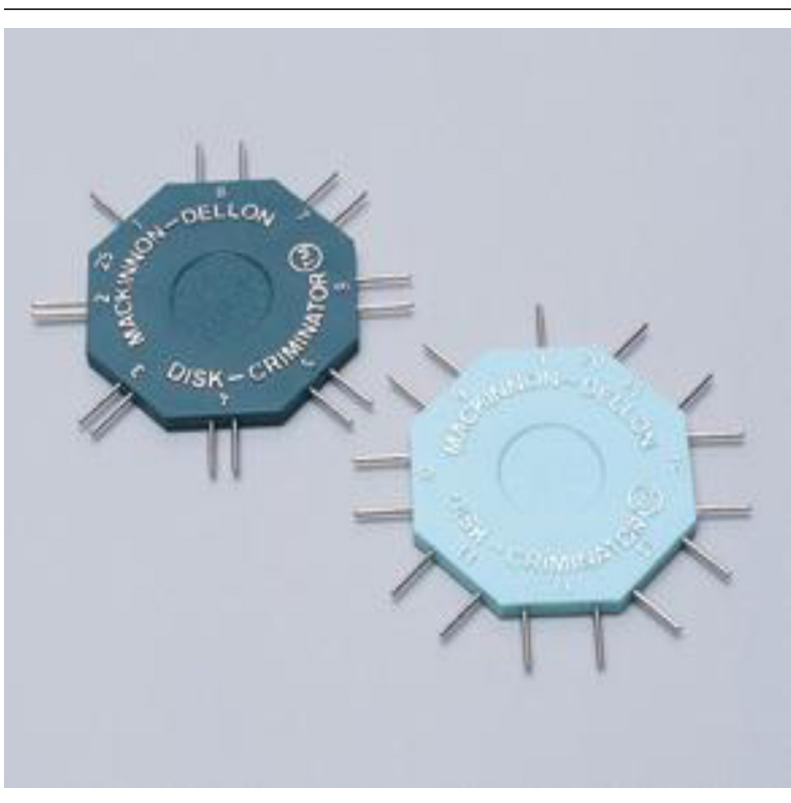

Figure 1. Dellon-McKinnon Disk-Criminator (Two-Point Discrimination Measurement Device)

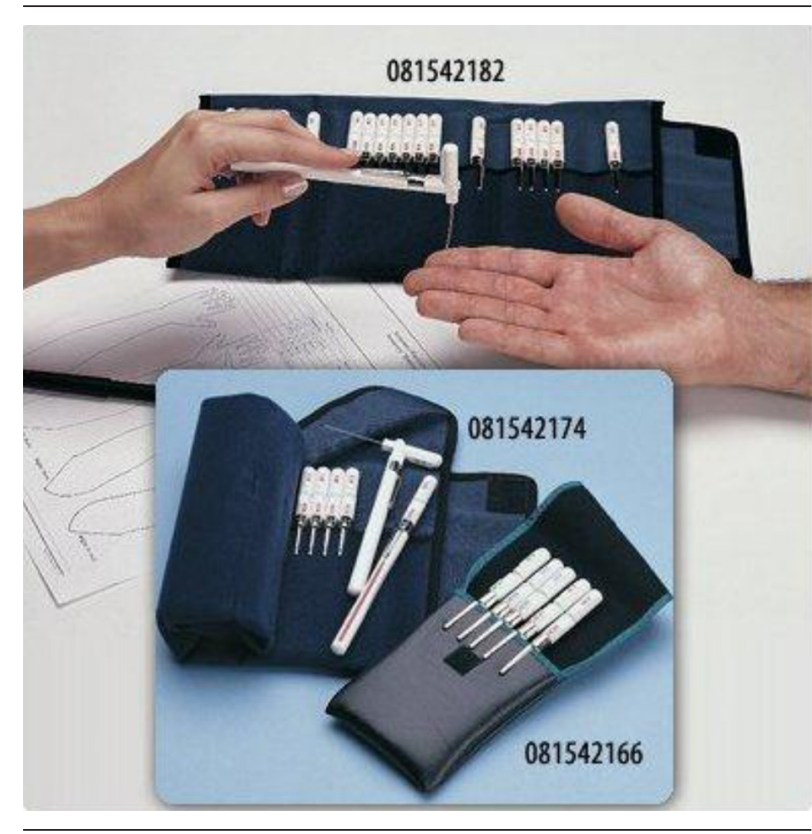

Figure 2. Light Touch Semmes-Weinstein Monofilament

If patients did not recognize two points, we repeated test with clips of larger distance.

To evaluate the light touch sense, we used SemmesWeinstein monofilament aesthesiometers (Semmes Weinstein Corp, Riverdale, New York, USA) with different thicknesses. These are calibrated, single-fiber, nylon threads identified by values ranging from 1.65 to 6.65 $\mathrm{mm}$, which generate reproducible bulky stress. The higher the value of the monofilament is, the stiffer and more difficult to bend it is. We used the filament with constant 
pressure to bend the filament on the skin of the healthy finger (Figure 2) (9).

If the patients recognized it, we would try the filament on the grafted skin; if they recognized it again, light touch threshold of the grafted finger would be recorded. If they did not recognize it, we would try another larger diameter filament until the patients could recognize it.

Ulcer on the skin graft has been evaluated by one surgeon, blinded to the cases, with direct observation of the fields for any fissures or epidermal separations that had led to exposed subcutaneous fat.

Contracture of the grafted skin was calculated by dividing the decrease in grafted skin size after 18 months by primary skin defect size (primary skin graft size). For measuring the grafted skin's size, we multiplied the maximum length by the maximum width of the grafted skin. For primary skin graft size, we calculated the defect size by the same method. After 18 months of follow-up, the percentage of the contracture was evaluated by the ratio of the grafted skin area decrease to primary skin graft area.

Hair on the grafted skin was evaluated by counting the hair follicles on the grafted skin.

Color difference between the grafted skin and recipient site was evaluated by analyzing the fingertip photographs on Photoshop CS5 (Adobe Sys Inc, San Jose, California, USA). The photographs were taken by a digital Canon camera (model IXUS800IS, Japan) in the same light and temperature condition. In this method, we evaluated Red (R), Green (G), and Blue (B) color histogram in ten spots of the grafted skin and recipient sites. Then the difference between mean scores of red, green, and blue were calculated as R, G, B; mean score of summation of G, B, and R was the final score for color difference $(C)$. The more $C$ was, the more color difference and less cosmetic result would be (Figures 3 and 4 ).

\subsection{Statistical analysis}

Data were collected in a questionnaire designed for this study and SPSS 15 was used to analyze the outcomes. Data were compared using independent samples t test or Mann-Whitney U test according to normal distribution of data on Kolmogorov-Smirnov test. P value $<0.05$ was considered statistically significant.

\section{Results}

The results of clinical evaluation of the fingertip skin grafts from the wrist and groin were compared in Figures 5 through 9. Except color difference variable, none of the other variables showed normal distribution; hence, we used Mann-Whitney U test to compare the results. Skin grafts in none of the fingers showed ulcer and none of the patients reported ulceration during the preceding 18 months. The grafted skin harvested from the volar wrist crease was called group 1 and that harvested from the groin was called group 2 .
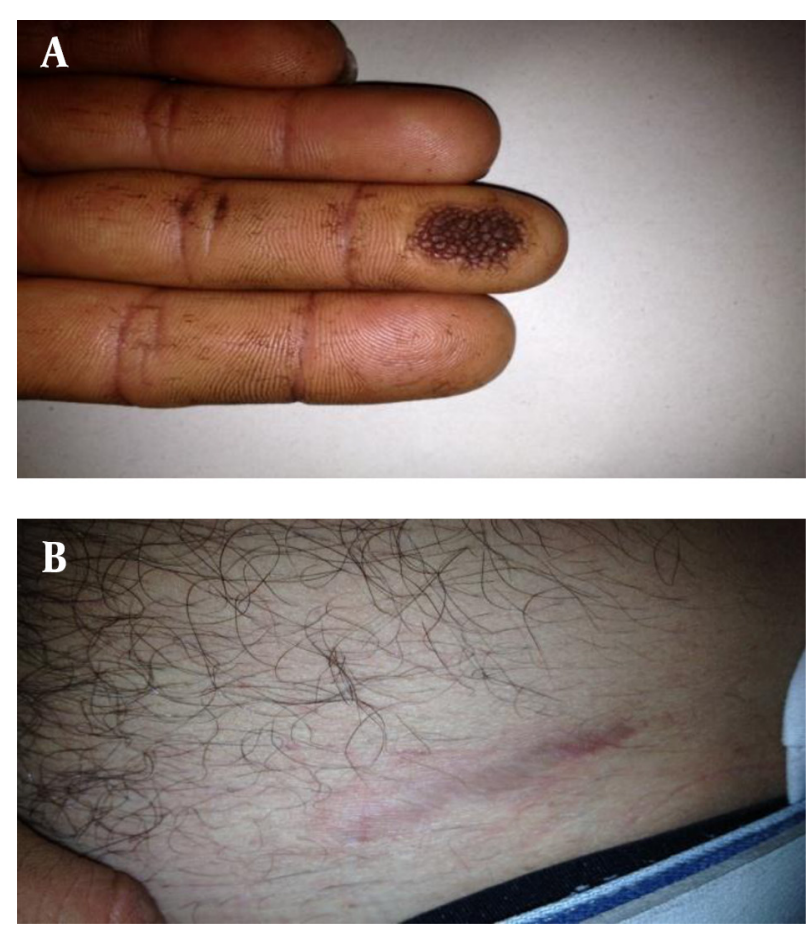

Figure 3. Skin Graft Harvested From Groin and the Result on Fingertip
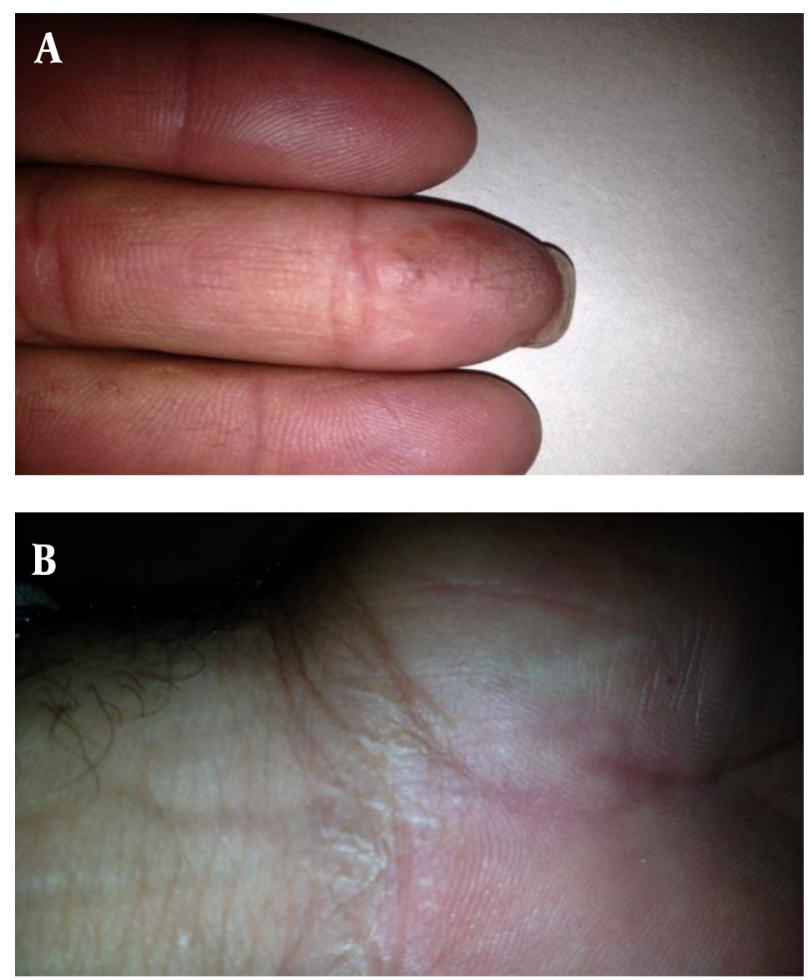

Figure 4. Skin Graft Harvested From Wrist and the Result on Fingertip 
Figure 5. The Comparison of Two-Point Discrimination Between Study Groups

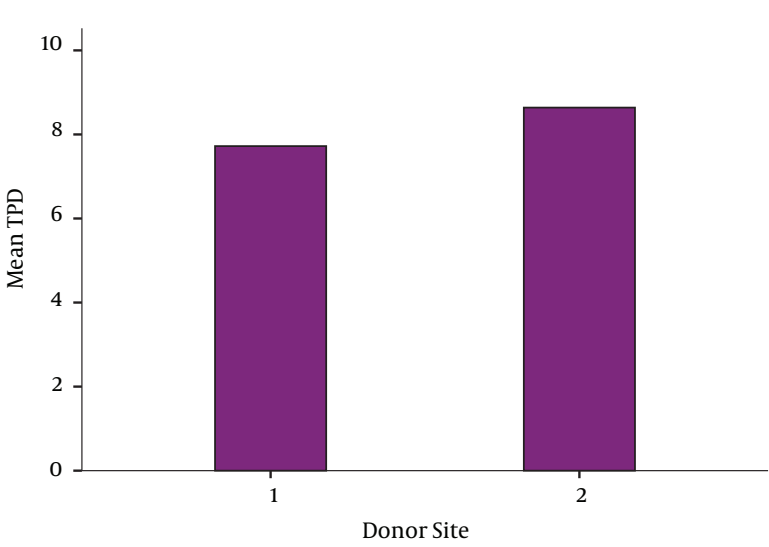

Skin graft was harvested from wrist in Group 1 and from groin in Group 2. $\mathrm{P}<0.05$ (Mann-Whitney U test as the variable was not normally distributed).

Figure 6. The Comparison of light Touch Between Study Groups

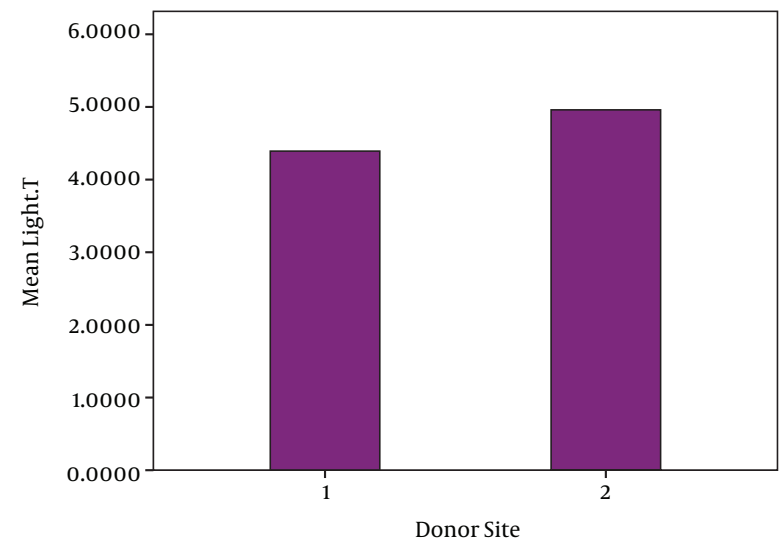

Skin graft was harvested from wrist in Group 1 and from groin in Group 2. $\mathrm{P}<0.05$ (Mann-Whitney $\mathrm{U}$ test as the variable was not normally distributed).

There was a significant difference between the two groups regarding the five mentioned characteristics including TPD, light touch, hair follicle counts, graft contracture, and color difference $(C)(P=0.000)$. The TPD mean score was $7.72 \pm 0.944 \mathrm{~mm}$ (range, 5-10 $\mathrm{mm}$ ) in group 1 and $8.64 \pm 0.867 \mathrm{~mm}$ (range, $7-10 \mathrm{~mm}$ ) in group 2 (Figure 5). The mean score of light touch was $4.39 \pm$ $0.409 \mathrm{~mm}$ (range, 3.84-5.07 $\mathrm{mm}$ ) in group 1 and $4.96 \pm$ $0.505 \mathrm{~mm}$ (range, $4.17-5.88 \mathrm{~mm}$ ) in group 2 (Figure 6).
Figure 7. The Comparison of Graft Contracture Percentage Between Study Groups

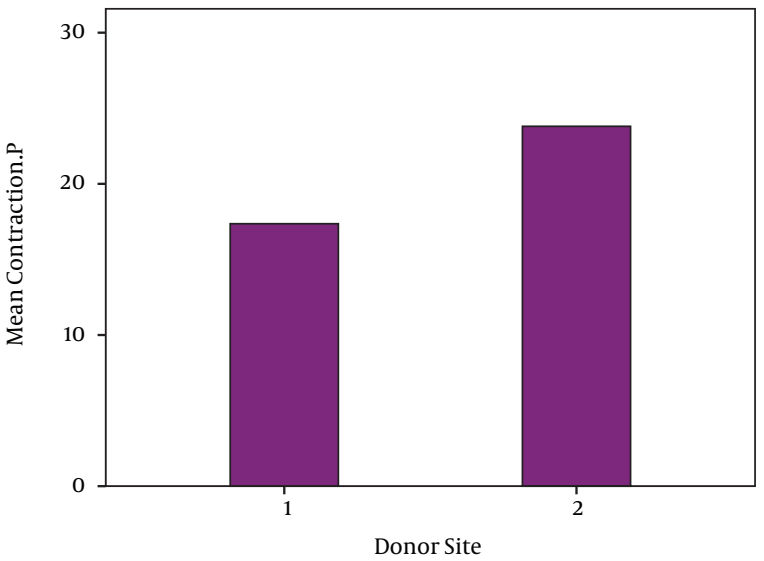

Skin graft was harvested from wrist in Group 1 and from groin in Group 2. $\mathrm{P}<0.05$ (Mann-Whitney $\mathrm{U}$ test as the variable was not normally distributed).

Figure 8. The Comparison of Hair Follicle Numbers Between Study Groups

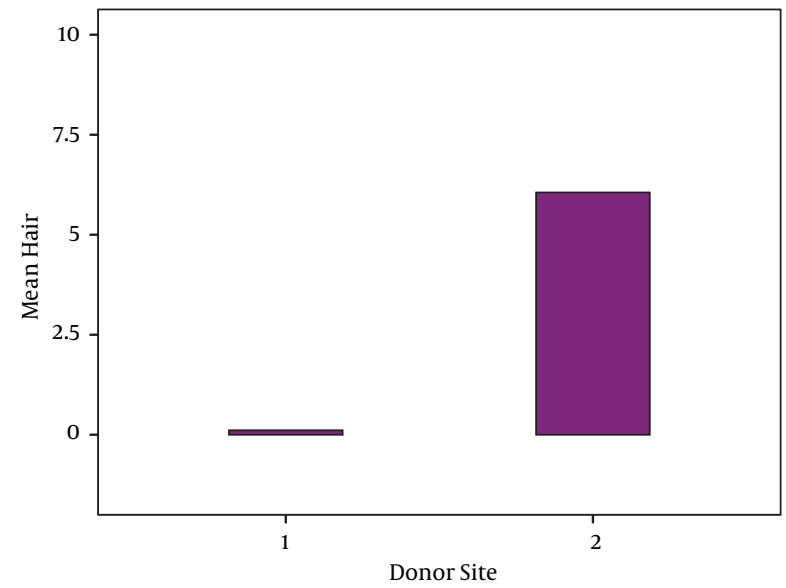

Skin graft was harvested from wrist in Group 1 and from groin in Group 2. $\mathrm{P}<0.05$ (Mann-Whitney U test as the variable was not normally distributed).

The mean score of the graft contracture percentage from the initial size was $17.36 \% \pm 3.091 \%$ (range, $10 \%$ $24 \%$ ) in group 1 and $23.81 \% \pm 2.539 \%$ (range, $19 \%-30 \%$ ) in group 2 (Figure 7 ). The mean number of the hair follicles was $0.11 \pm 0.398$ (range, $0-2$ ) in group 1 and $6.06 \pm$ 3.303 (range, 0-11) in group 2 (Figure 8 ). The mean score of graft color differences was $17.655 \pm 7.49$ (range, 3.436.2 ) in group 1 and $143.547 \pm 37.844$ (range, 101.5-248.1) in group 2 (Figure 9). 
Figure 9. The Comparison of Graft Color Difference with Recipient Site Between Study Groups

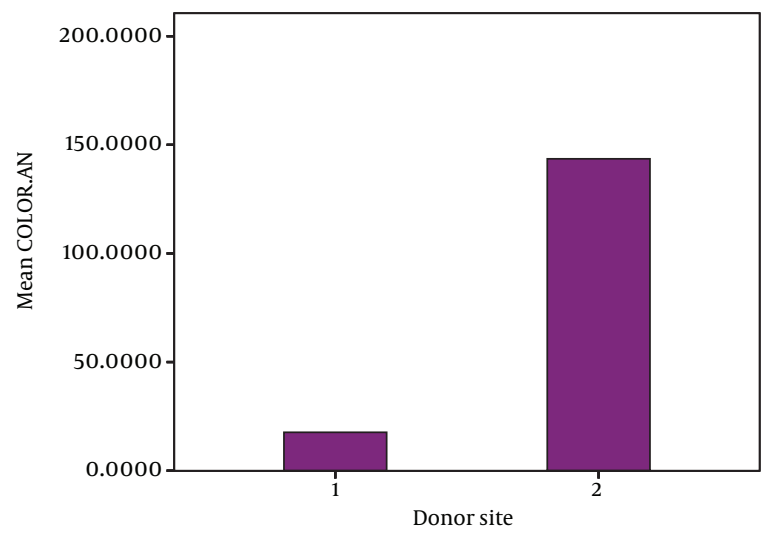

Skin graft was harvested from wrist in Group 1 and from groin in Group 2. $\mathrm{P}<0.05$ (independent-samples $\mathrm{t}$ test as the variable was normally distributed).

\section{Discussion}

The past few decades have witnessed significant advances in the treatment of fingertip injuries, but to the best of our knowledge, there has not been any comparative study on the clinical differences among donor sites of skin grafting. The current study compared the clinical result of two common donor sites of full-thickness skin grafts on the injured fingertips. Skin grafting is done in two popular ways: split thickness and full thickness. Split-thickness skin graft has epidermal and superficial dermal layer with 0.021 to 0.027 inch $(0.053$ to $0.068 \mathrm{~cm}$ ) thickness. Full-thickness skin graft has whole dermal and epidermal layer until 0.07 inch $(0.178 \mathrm{~cm})(1)$. Although hyperpigmentation and hair growth were found in most of the full-thickness skin grafts, full-thickness skin grafts have better sensory recovery (due to placement of sensory receptors at the deep dermis) and less contracture as well as breakdown in the fingertip skin; hence, it is the first choice when we have sufficient vascular bed at the receiver site $(3,10-17)$. On the other hand, full-thickness grafts do not survive as readily as split thickness grafts and require a much better bed to be placed on. When a full-thickness skin graft is used, the recipient area must be free of infection and homeostasis must work properly. Preferred donor areas for full-thickness skin grafts are the groin, volar aspect of the wrist crease, hypothenar region, cubital fossa, medial aspect of the arm, where the skin is thin and the created defect can be closed by undermining and suturing skin edges (18-23). The graft harvested from the wrist volar aspect is easier to harvest due to proximity to the recipient site. The only mentioned disadvantage for volar wrist crease skin graft is its scar which may be interpreted as suicidal attempt (1);nonetheless, the scar is minimal and approximately invisible in most cases.

In two studies, there was a correlation between fullthickness skin graft contracture and graft donor site $(12,24)$, which were the same as we experienced in our study, with the wrist region contracted meaningfully less than the groin region did. In other studies, the color difference between the grafted skin and recipient site and its correlation with sex, time of surgery, and donor site were analyzed. The results showed a stronger correlation with donor site and the time passed from the surgery $(19,20)$. Moreover, these results indicated that the volar wrist crease had the most compatible color resemblance with the fingertip skin compared to the groin region $(\mathrm{P}<$ 0.05), which were in accordance with the results of present study. However, we did not assess the effect of time on color change in long term, which could influence this comparison.

In our study, there was a significant difference in the five mentioned variables between the grafted skin harvested from the wrist and the groin $(\mathrm{P}<0.05)$.

One of the limitations to this study was short followup period, because longer follow-up could change the results, especially the color changes. We highly recommend larger sample-sized study to increase reproducibility, longer follow-up period, and comparing more donor regions to each other.

According to our findings, for fingertip injures that were larger than half of the distal phalanx pulp, full-thickness skin graft taken from the volar wrist crease had better clinical result including better TPD and light touch, less hair follicles on graft, less graft color difference with recipient site, and less graft contracture. Overall, the wrist skin graft possesses a number of advantages compared to groin skin graft. Therefore, the wrist-harvested skin graft is a high quality graft that can be considered as a good option to fulfill fingertip injury concern by a hand surgeon.

\section{Acknowledgements}

The authors express their thanks to all medical personnel of Orthopedic Surgery Ward in Nemazee and Imam Reza Hospitals for their cooperation. In addition, we would like to thank Dr. Nasrin Shokrpour for editorial assistance.

\section{Authors' Contributions}

Conception, study design, data collection, statistical analysis, drafting the manuscript, and critical revision of the manuscript for important intellectual content: Hamid Namazi, Reza Sharifzadeh, Behdad Eskandari Sani, and Farzam Mokarami; Study supervision: Hamid Namazi.

\section{References}

1. Green DP, Hotchkiss RN, Pederson WC, Wolfe SW. Green's Operative Hand Surgery. 5th ed. Philadelphia: Churchill Livingstone. 5 ed; 2005.

2. Canale ST, Beaty JH. Campbell's Operative Orthopaedics: Expert Con- 
sult Premium Edition-Enhanced Online Features.: Elsevier Health Sciences; 2012

3. Park YS, Lee JW, Huh GY, Koh JH, Seo DK, Choi JK, et al. , et al. Algorithm for Primary Full-thickness Skin Grafting in Pediatric Hand Burns. Arch Plast Surg. 2012;39(5):483-8.

4. Kim S, Chung SW, Cha IH. Full thickness skin grafts from the groin: donor site morbidity and graft survival rate from 50 cases. J Korean Assoc Oral Maxillofac Surg. 2013;39(1):21-6.

5. Tan E, Mortimer N, Salmon P. Full-thickness skin grafts for surgical defects of the nasal ala - a comprehensive review, approach and outcomes of 186 cases over 9 years. Br J Dermatol. 2014;170(5):1106-13.

6. Moghtadaei M, Farahini H, Faiz SH, Mokarami F, Safari S. Pain Management for Total Knee Arthroplasty: Single-Injection Femoral Nerve Block versus Local Infiltration Analgesia. Iran Red Crescent Med J. 2014;16(1).

7. Research Randomizer. Available from: http://www.randomizer org/form.htm.

8. Finnell JT, Knopp R, Johnson P, Holland PC, Schubert W. A calibrated paper clip is a reliable measure of two-point discrimination. Acad Emerg Med. 2004;11(6):710-4

9. Dros J, Wewerinke A, Bindels PJ, van Weert HC. Accuracy of monofilament testing to diagnose peripheral neuropathy: a systematic review. Ann Fam Med. 2009;7(6):555-8.

10. Lineaweaver WC. Skin graft coverage of critical marginal wounds in microsurgical cases. Microsurgery. 2013;33(4):315-7.

11. Sarifakioglu N, Terzioglu A, Bingul F, Aslan G. A hidden donor site for resurfacing finger tip defects: the 'ring graft'. Br J Plast Surg. 2003;56(8):797-803.

12. Bulic K. Long-term aesthetic outcome of fingertip reconstruction in complete syndactyly release. J Hand Surg Eur Vol. 2013;38(3):281-7.

13. Deunk J, Nicolai JP, Hamburg SM. Long-term results of syndactyly correction: full-thickness versus split-thickness skin grafts. J Hand Surg Br. 2003;28(2):125-30.
14. Chan QE, Barzi F, Harvey JG, Holland AJ. Functional and cosmetic outcome of full- versus split-thickness skin grafts in pediatric palmar surface burns: a prospective, independent evaluation. J Burn Care Res. 2013;34(2):232-6.

15. Davis W3, Wu C, Sieber D, Vandevender DK. A comparison of ful and split thickness skin grafts in radial forearm donor sites. $J$ Hand Microsurg. 2011;3(1):18-24.

16. Li WJ, Li C, Zhu J, Tian GL, Chen SL, Tian W. [Clinical study of fullthickness skin graft for reconstruction of completely defect nai unit]. Beijing Da Xue Xue Bao. 2012;44(6):860-5.

17. Yan H, Gao W, Li Z, Wang C, Liu S, Zhang F, et al. , et al. The management of degloving injury of lower extremities: technical refinement and classification. J Trauma Acute Care Surg. 2013;74(2):604-10.

18. Aasi SZ. Commentary: expanding the donor site options for fullthickness skin grafts. Dermatol Surg. 2010;36(4):532-3.

19. Takiwaki H, Miyaoka Y, Kohno H, Arase S. Graphic analysis of the relationship between skin colour change and variation in the amounts of melanin and haemoglobin. Skin Res Technol. 2002;8(2):78-83.

20. Kim JS, Park SW, Choi TH, Kim NG, Lee KS, Kim JR, et al. , et al. The evaluation of relevant factors influencing skin graft changes in color over time. Dermatol Surg. 2008;34(1):32-9.

21. Silapunt S, Peterson SR, Alam M, Goldberg LH. Clinical appearance of full-thickness skin grafts of the nose. Dermatol Surg. 2005;31(2):177-83.

22. Paletta C, Pokomy J, Rumbolo P, Mathes S. Mathes Plastic Surgery. 2 ed: Philadelphia: Saunders; 2006.

23. Kaltman JM, McClure SA, Lopez EA, Pedroletti F. Closure of the radial forearm free flap donor site defect with a full-thickness skin graft from the inner arm: a preferred technique. J Oral Maxillofac Surg. 2012;70(6):1459-63.

24. Iwuagwu FC, Wilson D, Bailie F. The use of skin grafts in postburn contracture release: a 10-year review. Plast Reconstr Surg. 1999;103(4):1198-204. 